\title{
L'écriture des adolescents : une démarche ethnographique pour rendre compte de la spatialisation des pratiques
}

\section{Élisabeth Schneider}

\section{(2) OpenEdition \\ Journals}

Édition électronique

URL : http://journals.openedition.org/cdg/2346

DOI : $10.4000 /$ cdg. 2346

ISSN : 2107-7266

Éditeur

UMR 245 - CESSMA

Référence électronique

Élisabeth Schneider, «L'écriture des adolescents : une démarche ethnographique pour rendre compte de la spatialisation des pratiques », Carnets de géographes [En ligne], 3 | 2011, mis en ligne le 01 décembre 2011, consulté le 07 mai 2019. URL : http://journals.openedition.org/cdg/2346 ; DOI $10.4000 /$ cdg. 2346

\section{(c) (i) $\odot$}

La revue Carnets de géographes est mise à disposition selon les termes de la Licence Creative Commons Attribution - Pas d'Utilisation Commerciale - Pas de Modification 4.0 International. 


\title{
L'ECRITURE DES ADOLESCENTS :
}

Une démarche ethnographique pour rendre compte de la spatialisation des pratiques

\author{
ÉLISABETH SCHNEIDER \\ Université de Caen Basse-Normandie \\ Laboratoire ESO-Caen \\ Géographie et sciences de l'information et de la communication \\ elisabeth.schneider@unicaen.fr
}

\section{Résumé}

Ces réflexions méthodologiques s'inscrivent dans le cadre d'un travail de thèse sur les pratiques d'écriture des adolescents et leur spatialisation organisant un paysage scriptural complexe. La complexité et la richesse de ces pratiques spatiales renvoient d'une part, à la question de la production de la réalité sociale, élaboration discursive et concrétisation de systèmes symboliques, et d'autre part, à la réflexion sur la production de connaissances permise et contrainte par le dispositif d'enquête. En restituant une pratique de terrain, on cherche à montrer que l'écriture dans la recherche donne à voir son objet et que les questions soulevées émergent de la description et ne sont pas préexistantes. De la même façon, les écrits produits par les adolescents, leur circulation, leur dissémination jouent un rôle performatif dans leur expérience et la production des espaces.

\footnotetext{
Abstract

These methodological considerations are part of a thesis on the writing practices of teenagers and their spatial organization of what we choose to call "a complex scriptural landscape". The complexity and the richness of these spatial practices refer to the question of the production of a social reality, elaboration of discursive practices and concretization of symbolic systems. On the other side, it highlights that the production of knowledge in research is both made possible and constrained by the survey plan. Based on a reflection about the fieldwork, we aim to show that writing in the context of the research creates, in a certain way, its purpose and that the issues are emerging from the description rather than being pre-existing. In the same way, the writings produced by teenagers, the circulation of these writings, and their dissemination play a performative role in the experience and the production of spaces by young people.
} 


\section{Introduction}

Ces réflexions méthodologiques s'inscrivent dans le cadre d'un travail de thèse sur les pratiques d'écriture des adolescents et leur spatialisation organisant un paysage scriptural complexe. On dira cette écriture " outillée " dans la mesure où on étudie la place des artefacts dans l'économie scripturale des adolescents, du papier au numérique. Les adolescents actuels sont nés dans une période de développement des technologies de l'information et de la communication. Ceux que l'on appelle, depuis une dizaine d'années, les "digital natives ${ }^{1}$ ", déclarent un grand nombre de pratiques et semblent en maîtriser les outils. Ils sont aussi scolarisés et formés à des pratiques de l'écrit sur papier depuis leur entrée à l'école. Dans les espaces produits et vécus par les adolescents, quel est le rôle aujourd'hui de l'écriture? Pour en percevoir la densité et la diversité, j'ai adopté une démarche ethnographique que j'ai construite en m'appuyant sur des éléments méthodologiques mis en évidence par certains travaux en ethnologie et géographie (Beaud, Weber, 1997, 2008, Crang, 2003 ; Danic, David, Depeau, 2010 ; Danic, Delalande, Rayou, 2006) mais aussi en travaillant à des spécificités de l'observation auprès d'adolescents soucieux de se préserver du regard des adultes. Comment accéder à la réalité de ces pratiques adolescentes, à savoir par exemple les écrits entre pairs, hors et dans la classe, sur les réseaux sociaux numériques qu'ils fréquentent ? Comment comprendre l'agencement spatial en œuvre et choisir les situations vécues par ces adolescents qui permettront de mettre en évidence le mode de relation qu'ils entretiennent à l'espace et le rôle que l'écriture y joue? Quelle articulation à la question du contrôle symbolique et technique dont l'institution scolaire, mais aussi les technologies relationnelles mises en œuvre par les réseaux numériques, sont des instances?

La complexité et la richesse de ces pratiques spatiales renvoient d'une part, à la question de la production de la réalité sociale, élaboration discursive et concrétisation de systèmes symboliques, et d'autre part, à la réflexion sur la production de connaissances permise et contrainte par le dispositif d'enquête.

\section{Premiers jalons du dispositif d'observation ethnographique}

Le recueil de données a été voulu le plus diversifié possible et adapté à ce qui paraissait être caractéristique de l'expérience adolescente (Rayou, 2001 ; Pasquier, 2005). J'ai choisi de suivre des adolescents dans leurs déplacements et leurs activités, dans la sphère scolaire et privée, mais aussi de collecter tous les écrits produits par les adolescents, quels qu'ils soient. L'objectif est ensuite de les inscrire dans un contexte de production et d'entendre ce que ces adolescents ont à en dire pour percevoir la complexité et le rôle de ces écrits dans la production des espaces.

Les premiers contacts ont été alternativement informels et sous forme d'entretiens, auprès de deux lycéennes. Puis, suite à leur sollicitation, j'ai rencontré huit garçons et filles de seconde. Ces moments répondaient à l'objectif d'établir des relations avec eux

\footnotetext{
${ }^{1}$ Par opposition aux digital immigrants (Prenski, 2001), les 13-18 ans seraient doués d'une appétence et de compétences quasi-naturelles à l'usage des Technologies numériques, ce qu'un certain nombre de recherches interrogent, voir les travaux d'E. Harguittaï (2010).
} 
et d'explorer des pistes pour déterminer les observations nécessaires à mener par la suite: quels lieux? A quels moments? La difficulté était de trouver des modalités d'observation permettant la prise en compte de la complexité de la production d'espaces telle qu'elle a été mise en évidence par M. Lussault (Lussault, 2000, 2007) 2 . Les adolescents sont producteurs d'espaces sociaux et sont aussi insérés dans les espaces produits par d'autres. Mais comment rendre compte de l'hétérogénéité et cependant possible continuité de cet ensemble pratique ${ }^{3}$ ? J'ai choisi certaines situations fréquemment vécues par les adolescents et qui condensaient leurs relations à l'espace par et avec l'utilisation de l'écriture. Le cours en classe de lycée, occupant ainsi une place essentielle en termes de durée dans l'expérience adolescente, a fait l'objet de nombreuses heures d'observation.

Un premier entretien collectif élargi a donc eu lieu avec huit lycéens ${ }^{4}$ dans un lieu que je qualifierais de " semi-public » et qu'ils ont proposé: il s'agissait de la partie thé-café d'un bouquiniste. Pour commencer cet entretien d'une heure, j'ai précisé ce que je souhaitais, à savoir observer ce qu'ils font, la réalité de leurs pratiques sans jugement de valeur, ni scolaire ni privé, et que ce que je verrai serait systématiquement anonymisé, qu'aucun compte-rendu de ce que je pourrais voir ne serait fait aux parents ni aux enseignants sans passer par le filtre du travail d'écriture de recherche, qu'ils n'avaient donc à craindre aucun retour négatif pour des écrits, attitudes transgressives de la norme scolaire en particulier. Ma volonté de restituer la réalité de leurs pratiques est un élément qui "compte» pour eux, c'est celui que les uns après les autres, ils utilisent pour me présenter ensuite à leurs camarades. Dans le premier entretien, les deux adolescentes déjà interviewées ont à plusieurs reprises encouragé les autres à me raconter et à me montrer ce qu'ils faisaient relativement à l'écriture et qui touchent à des éléments très personnels, puisqu'il peut s'agir aussi bien de SMS, de notes de cours, que de mots écrits pendant les cours ou de fil de commentaires sur Facebook.

J'ai rencontré un des professeurs principaux à qui j'ai présenté mon projet et qui l'a relayé sous forme de courrier à l'ensemble de ses collègues pour que je puisse assister aux cours; insistant sur le fait que ce sont les pratiques des adolescents qui m'intéressent et non les pratiques enseignantes. Ce temps de prise de contact fut essentiel et permit d'expliciter mon projet, d'instaurer une confiance avec les enseignants et leur assurer que je ne viens pas pour "espionner» ou "critiquer»; on me précise que le nom de mon directeur de thèse et le fait que je ne sois pas trop jeune rassurent. Ce lycée d'enseignement général de 1400 élèves, situé au centre d'une grande ville mais dont le secteur de recrutement s'étend à une trentaine de kilomètres à l'extérieur et dont la population scolaire est hétérogène, a accepté de m'accueillir sur des demi-journées ou journées pour que je puisse suivre les élèves dans leurs classes.

\footnotetext{
${ }^{2}$ Selon Lussault, l'espace de la situation est le résultat de la combinaison de trois plans: le cadre matériel, le déroulement qui fonde l'agencement spatial et le mode de relation pratique et idéel que chaque individu instaure à l'espace. Les outils de l' «écrire» peuvent être ceux d'un agir spécifique des adolescents.

3 "Ensemble pratique " se comprend ici au sens de Michel Foucault, comme ce que font les individus et la façon dont ils le font. Le philosophe s'y intéresse comme domaine homogène de référence qui permet de penser les formes de rationalité qui organisent les manières de faire.

${ }^{4}$ Ces premiers entretiens ont été un préalable essentiel pour démarrer les observations en classe puisque c'est ce qui a déterminé le proviseur à accepter de me rencontrer.
} 


\section{Complexité de l'observation en classe}

\section{Qu'est-ce qu'une classe?}

La classe est ainsi une constante de l'univers scolaire mais qui recouvre des réalités hétérogènes. C'est a minima un endroit clos dans lequel se joue une situation scolaire. C'est aussi un groupe d'élèves, auquel l'institution peut donner arbitrairement une identité, mais qui est un groupe social avec ses dynamiques et ses logiques. L'organisation des programmes d'enseignement du lycée en groupes et modules, par le jeu des options, fait que l'on a parfois à faire à une classe précise, parfois à des groupes d'élèves appartenant à différentes classes. Les dimensions de la production d'espaces par les adolescents se font de manière complexe dans des configurations dynamiques et évolutives, dans la mesure, aussi, où leurs pratiques s'inscrivent dans des salles de classe organisées dans un certain nombre de $\mathrm{m}^{2}$, avec du mobilier, des affichages, et symboliquement par des organisations de disciplines scolaires (travail de groupe, place de l'oral et des interactions, rôle des outils qui médiatisent le savoir). Certaines salles de classe permettent un déplacement fréquent, en arts plastiques par exemple, d'autres sont relativement petites et toutes les chaises sont occupées. Certains dispositifs spatiaux ne pas toujours en lien avec la discipline du cours: laboratoire et "paillasses» sont prévus pour des cours de sciences mais accueillent aussi des cours de français. Ces différentes dimensions élaborent une réalité complexe: l'agencement matériel du lycée manifeste ainsi un système symbolique de la représentation du savoir, du rapport à la discipline, celui de la forme scolaire portée par l'institution, mais traduit aussi les sédimentations des organisations scolaires successives. Tout cela se concrétise à différents niveaux: la salle de classe, selon la discipline à l'usage de laquelle elle est affectée, rend visible la représentation du savoir de celle-ci mais d'autres systèmes symboliques se juxtaposent et/ou s'articulent au premier : celui de l'enseignant, celui des adolescents qui par leur appropriation des lieux et les choix d'agencement qu'ils font, peuvent instaurer d'autres systèmes signifiants. Ces systèmes sémantisent le réel par des productions discursives, et font advenir les espaces sociaux (Chivallon, 2008).

\section{Prendre le temps}

Au cours des premières heures d'observation, la priorité a été donnée à la visibilité du chercheur et à l'instauration de relations de confiance avec les élèves et les adultes selon des modalités différentes. Dans les premiers moments, les observations ont été minimales pour permettre l'installation d'une routine de la présence du chercheur. Petit à petit, j'ai pu photographier des tables comportant un certain nombre d'artefacts de l'écriture, après avoir demandé l'autorisation aux élèves concernés. Ils s'écartent de leur table pour me permettre de le faire puis se remettent à leur tâche, parfois montrent leur étonnement de mon intérêt pour des éléments si quotidiens. L'ensemble des observations respecte une sorte de protocole, aux étapes signifiantes qui correspondent à des seuils symboliques: établir/rétablir le contact avec les élèves par l'envoi d'un SMS quelques heures avant la rencontre, entrer en contact, verbal ou non-verbal, avec ceux qui attendent dans le couloir, avec l'enseignant, entrer dans la classe et choisir sa place, signifiante dans la mesure où elle doit être un poste d'observation, mais aussi un point de visibilité de ce que fait le chercheur pour les 
autres acteurs sans gêner le travail scolaire. Chaque nouveau temps d'observation amène à reposer la question de mon attitude, de ma place dans la classe en tant que groupe symbolique et en tant que lieu: où m'asseoir? Puis-je me déplacer? Quelle attitude renvoyer à l'enseignant? Comment regarder et garder trace des pratiques sans être trop intrusive? Dans quelle mesure suis-je aussi intégrée dans des espaces que j'observe? Soigner les détails rendant visible la confidentialité et la congruence entre mes attitudes et mes demandes permet d'instaurer la confiance et de la maintenir mais cela contribue aussi à la production d'un système de significations dont on ne peut être totalement maître.

\section{L'observateur aux prises avec les processus de spatialisation}

\section{Une réflexivité nécessaire 5}

Cette dimension de réflexivité a été particulièrement travaillée ces dernières années par des recherches anglo-saxonnes et féminines et renouvellent la réflexion du chercheur comme acteur social situé, souhaitant mener une recherche éthique, en particulier, concernant la relation enquêteur/enquêté. La recherche est, en effet, nécessairement incarnée (Crang, 2003): dans mon cas, j'ai plusieurs statuts différents : professeure dans le second degré, formatrice d'enseignants, intervenante associative auprès d'adolescents et mère d'adolescents. Observatrice ponctuelle, j'ai une position différente en fonction des interlocuteurs: les élèves m'appellent «la dame de l'université» et les enseignants la "prof qui fait une thèse». Mon statut de professeure induit avec ces derniers une forme de connivence culturelle, concernant en particulier les difficultés posées par la gestion de l'autorité. Je dois démêler ces aspects dans mes observations et mes analyses pour un travail d'objectivation (Weber, 2009). Dans quelle mesure ma connaissance des adolescents/élèves joue un rôle dans mes hypothèses et ma manière d'observer? L'expérience de l'école, mon regard sur les adolescents/élèves, fruit d'un processus de médiation sociale et culturelle, s'est construit au fil de mon passé professionnel. Mes activités associatives ont permis de le distancer mais sont à l'origine d'un postulat très net dans mon travail: les adolescents ont des pratiques beaucoup plus riches qu'ils ne le montrent en contexte scolaire, richesse que l'école et eux-mêmes contribuent à cacher pour des raisons variées. C'est ce qui me conduit à prendre au sérieux la parole adolescente ${ }^{6}$. Parallèlement, la situation qui se crée ainsi doit être analysée et réfléchie: le leurre d'une communication transparente est un écueil réel. La confidentialité est une nécessité pour permettre la parole et accéder aux pratiques mais elle devient une forme de connivence complexe: le chercheur observe les pratiques au plus près mais n'est pas un adolescent. Comment les adolescents interprètent-ils le fait qu'un adulte observe leurs pratiques transgressives et ne les relève pas, voire les encourage puisqu'elles sont les traces recherchées? Quel est l'impact de cette observation sur leurs propres pratiques? Une lycéenne m'a par exemple renvoyé des SMS pendant le cours et qui sont les traces

\footnotetext{
${ }^{5}$ On prendra pour exemple, F. Sultana en 2007 dans le cadre d'une recherche sur l'accès à l'eau au Bangladesh, qui montre par exemple à quel point les indices physiques rendent visible son étrangeté aux enquêtées et font l'objet d'une analyse pour le travail de terrain dans la mesure où elles modifient les situations observées.

6 Sans pour autant nier la part «d'opacité et de préreflexivité» que peut receler le langage de l'acteur (Lussault, $2000: 29$ )
} 
d'une conversation qu'elle mène avec des amis dans un autre lycée. N'est-ce pas une intervention modifiant les paramètres de l'observation? Cette question se pose mais différemment sur les plates-formes de jeux de rôle, les forums, les réseaux sociaux tels que Facebook dans la mesure où d'une part, une quasi invisibilité est possible ${ }^{7}$ et d'autre part, le fait que l'on soit dans un cadre asynchrone et distant, qui donne plus d'autonomie dans les collectes de données. C'est pour cela qu'il est important d'articuler les paroles recueillies lors des entretiens avec des écrits composés en ma présence, hors de ma présence, et avec les observations. Par ailleurs, la recherche évolue aussi du fait que les enseignants proposent d'enquêter dans certaines classes, avec des collègues qui ont des pratiques spécifiques de l'écrit en contexte scolaire et qu'ils ouvrent des pistes de réflexion permettant à l'apprentie-chercheur de se décentrer de sa perception de l'objet.

\section{Démarche épistémologique et terrain}

Des processus spécifiques de spatialisation et de médiation socioculturelle sont en jeu sur les réseaux sociaux, dans des espaces privés hors des temps scolaires et entrent en relation avec ce qui se joue au lycée, ce qui nécessite de collecter des données sur ces différents espaces. Cet ensemble composite demande de problématiser la pratique du terrain (Calberac, 2011). Aux observations en classe s'ajoutent des observations sur le réseau internet par le biais de captures d'écrans régulières des espaces d'écriture de ces mêmes adolescents. La prise en compte de l'objet technique instrumentant l'écriture est nécessaire dans la mesure où cette dernière se déploie aujourd'hui sous des formes extrêmement variées: écriture scolaire (dans ou hors l'école) et écriture privée (aux modalités, finalités et supports divers: conversation sur Facebook ou roman pour soi). Le nomadisme et la facilité d'utilisation des outils numériques amènent l'écriture dans des lieux où elle semblait quasiment exclue, comme les transports en commun et interrogent en cela l'appropriation des lieux où les adolescents séjournent ou qu'ils traversent. Ces lieux sont aussi un lieu d'observations, où je garde mon carnet à portée de main. Le carnet permet de garder la trace de données factuelles et de pistes d'analyse qui permet ensuite une écriture descriptive dense et documentée (Geertz, 1998). Les hypothèses se construisent à partir des données brutes et du travail d'écriture du carnet de terrain, ce qui permet une objectivation heuristique, englobant l'espace de recherche singulier ${ }^{8}$ produit par la pratique de recherche même.

Que dégager de ces observations sur l'écriture comme facteur et outil de production d'espaces? L'écriture outillée par le numérique apparaît bien comme un outil de la production et gestion des espaces et soulève la question d'une métrique spécifique pour organiser les distances: envoyer des SMS pendant un cours actualise une coprésence entre pairs distants et produit un espace social «à côté» d'espaces

\footnotetext{
${ }^{7}$ Mon nom apparaît dans les listes d'amis, mais je travaille «hors-ligne», ainsi les adolescents ne me voient pas présente sur leur espace et je n'interviens jamais, afin que, techniquement, aucun lien provoqué par moi ne s'établisse entre des réseaux d'adolescents. Dans les entretiens, plusieurs disent avoir «oublié» qu'ils m'avaient invitée.

${ }^{8}$ A partir de la notion d'acteur-réseau de Latour, Calberac montre que le chercheur inscrit l'espace de recherche dans un réseau dont «chaque nœud [sur lequel on porte l'attention en retour] modifie l'espace étudié» et permet le déploiement de l'interprétation. http://www.carnetsdegeographes.org/PDF/terrain_02_01_Calberac.pdf [p.4]
} 
matérialisés par d'autres écrits: feuille de classeur, tableau dont on recopie ou pas les données, petit mot que l'on fait circuler... Par ailleurs, l'écriture manuscrite et électronique, semble être un outil et une ressource de production de l'espace social. En effet, la réalité sociale de ces adolescents, matérialisation d'un système symbolique et d'une imagination à l'œuvre (Chivallon, 2008), est une élaboration langagière dont les technologies outillant l'écriture permettent le déploiement. Ainsi, dans une des classes observées, une $\mathrm{BD}$ se fabrique en passant d'un élève à l'autre et double l'activité scolaire et sa temporalité de la production d'un espace invisible à l'adulte, qui permet de donner sens à l'événement du cours, crée une médiation de la réalité et réorganise la temporalité (Crang, 1999). Dans une contribution sur l'usage des images dans les pratiques touristiques, $M$. Crang étudie précisément le " faire » des touristes quand ils photographient des sites: la trace, par l'image, crée l'expérience. Comme artefact permettant la médiation, certains parallèles peuvent être dégagés pour analyser les écrits des adolescents.

Figure 1 : BD racontant une journée de lycée et ses imprévus, élaborée par des élèves de seconde. Elle fut dessinée en cours puis partagée sur Facebook.

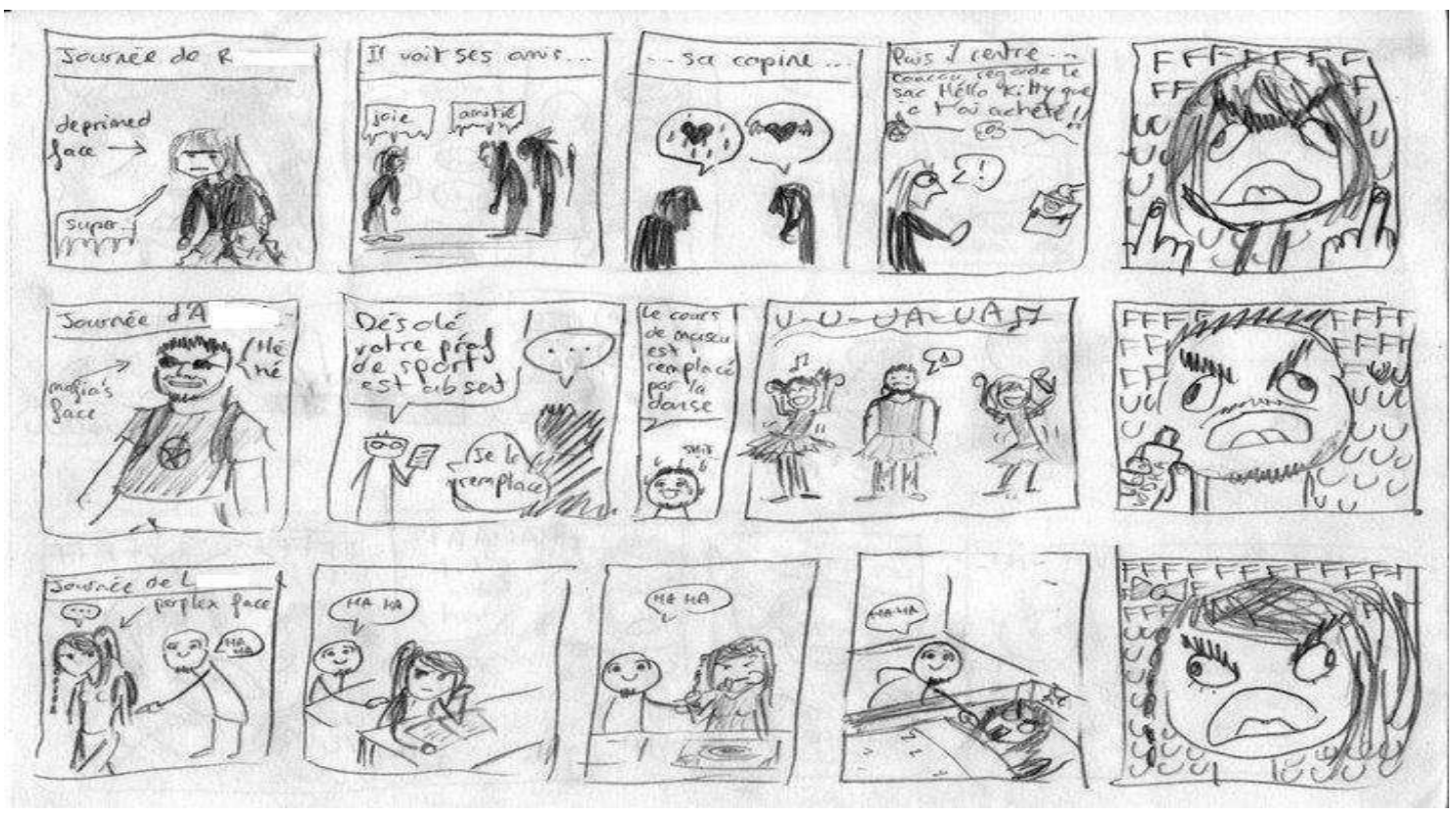

L'écriture est omniprésente dans les pratiques adolescentes, alors que les enseignants insistent sur la réduction de celle-ci. Le partage visibilité/invisibilité des pratiques qui semble s'organiser, soulève le problème suivant: l'écriture, outil de l'ordre scolaire, support de savoir, mode de restitution, outil de vérification et d'évaluation, donc du côté de la stratégie (De Certeau, 1990) semble aussi être un outil d'émancipation, de tactique possible pour les adolescents. 


\section{Conclusion}

La restitution d'une pratique de terrain a permis de montrer que le processus d'élaboration cognitive de la recherche (Cefaï, 2003) est performatif dans la mesure où l'écriture de recherche (associée ici à un corpus d'images qui redouble la question de la médiation) donne à voir son objet. En outre, les questions soulevées émergent de la description et ne sont pas pré-existantes. Les écrits produits par les adolescents, leur circulation, leur diffusion dans les différents espaces et leur performativité pour faire advenir l'expérience même constituent des axes importants issus de cette pragmatique de la recherche. Les travaux dirigés par Daniel Fabre en anthropologie de l'écriture ont montré le lien entre écritures ordinaires et identité pour les adolescents (Fabre, 1997). On peut supposer que la prise en compte de la contribution de l'écriture à la production d'espaces renouvelle cette question de l'identité adolescente, en particulier concernant les processus de médiatisation de soi, de production du réel et de l'organisation de l'expérience. Quels sont les principes organisateurs de la production de ces espaces, entre, par exemple, la dimension institutionnelle de l'école et la volonté des adolescents d'être visible/invisible des pairs, des adultes? Quelles compétences sont mises en œuvre pour gérer, en particulier, les distances dans l'élaboration de ce tissu spatial? Comment coexistent et se recoupent ces espaces? Quelle élaboration discursive se fait jour pour donner sens et continuité à l'expérience adolescente? Ce sont quelques questions qui pourront à leur tour nourrir la pragmatique de la recherche.

\section{Bibliographie}

CALBERAC Y., (2011), "Le terrain des géographes est-il un terrain géographique ? Le terrain d'un épistémologue», in Carnets de géographes, ${ }^{\circ}{ }^{\circ}$, mars 2011 . Accessible en ligne: http://www.carnetsdegeographes.org/PDF/terrain 0201 Calberac.pdf

CEFAÏ D. (2003), «Postface : L'enquête de terrain en sciences sociales», in L'enquête de terrain, textes réunis, présentés et commentés sous la direction de Daniel Céfaï, Paris, La Découverte, MAUSS, pp.465-615.

CHIVALLON C., (2008), "L'espace, le réel et l'imaginaire : a-t-on encore besoin de la géographie culturelle? ", in Annales de géographie, Paris: Armand Colin, pp. 67 à 89.

CRANG, M. (2003), "Qualitative methods: touchy, feely, look-see?", in Progress in human geography., 27 (4). pp. 494-504.

CRANG M., (1999), "Knowing, Tourism and practices of vision", in Crouch, (1999), Leisure, tourism Geographics practices and geographical knowledge, Londres: 
Routhage, pp.238-256.

DANIC I., DAVID O., DEPEAU S., (2010), Enfants et jeunes dans les espaces du quotidien, Rennes, PUR. 274 p.

DANIC I., DELALANDE J., RAYOU P., (2006), Enquêter auprès d'enfants et de jeunes, objets, méthodes et terrains de recherche en sciences sociales., Rennes, PUR. 216p.

CERTEAU (de) M., (1990), L'invention du quotidien, 1. Arts de faire, Paris: Gallimard, p. 147 et suivantes.

FABRE D., (dir.), (1997), Par écrit: ethnologie des écritures quotidiennes, (Textes réunis par Martin de la Soudière et Claudie Voisenat), Paris, éditions de la MSH, $394 p$.

FOUCAULT M., (1984), "Qu'est-ce que les Lumières ? ", in Dits et écrits, (1994), Paris, Gallimard, vol. IV, p. 562-578.

GEERTZ C., (1 ${ }^{\text {ère }}$ édition 1998), La description dense : vers une théorie interprétative de la culture, in L'enquête de terrain, (2003), textes réunis, présentés et commentés sous la direction de Daniel Céfaï, Paris, La Découverte, MAUSS, pp. 208-233.

HARGITTAI, E. (2010). Digital Na(t)ives Variation in Internet Skills and Uses among Members of the "Net Generation". Sociological Inquiry. 80(1), pp.92-113.

LUSSAULT M., (2000), Action(s)! in Lussault M., Lévy J., (dir), Logiques de l'espace, esprit des lieux, Géographies à Cerisy, Paris, Belin, pp.11-36.

LUSSAULT M., (2007) L'homme spatial. La construction sociale de l'espace humain, Paris, Seuil, 366 p.

PASQUIER D., (2005), Cultures lycéennes: la tyrannie de la majorité, Paris, éd. Autrement, 180p.

PRENKSY, M. (2001a). « Digital Natives, Digital Immigrants. », On the Horizon, 9(5).

RAYOU P., (2001), La cité des lycéens, Paris, L'Harmattan, 295p.

SULTANA F., (2007), "Reflexivity, Positionality and Participatory Ethics: Negotiating Fieldwork Dilemmas in International Research», in ACME: An International E-Journal for Critical Geographies, 6 (3), pp.374-385.

WEBER F., (2009), De la modélisation à la description armée: le cas de l'ethnographie réflexive, Manuel de l'ethnographe, Paris, PUF, pp.125-135. 